
| |

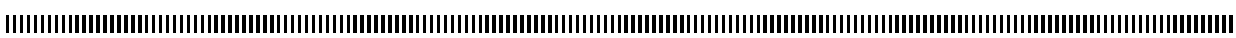

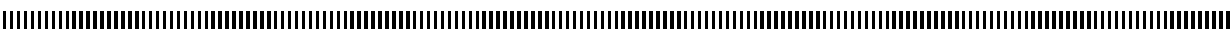

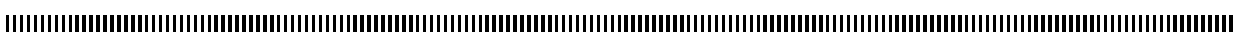
| |

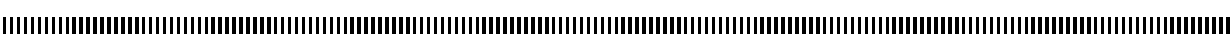

Iterative solvers

\title{
Numerical study of some iterative solvers for acoustics in unbounded domains
}

\author{
Nabil Gmati* - Naouel Zrelli** \\ Laboratoire LAMSIN \\ Ecole Nationale d'Ingénieurs de Tunis \\ B.P. 37,1002 Tunis \\ TUNISIE \\ * nabil.gmati@ipein.rnu.tn \\ ** naouel.zrelli@enit.rnu.tn
}

|

RÉSUMÉ. L'objectif de cet article est de présenter et d'étudier quelques méthodes itératives, utilisant les méthodes de décomposition de domaine pour la propagation d'ondes acoustiques harmoniques en domaine extérieur. On développe notre méthode dans le cas d'un guide infini dans une direction et celui du problème de diffraction par un obstacle. Dans les deux cas, on utilise des conditions aux limites transparentes connues, qui imposent sur une frontière fictive une condition aux limites utilisant un développement en série de Fourier. En vue de la mise en œuvre numérique, on propose un algorithme original, obtenu en appliquant la méthode des itérations successives au problème posé dans le domaine tronqué. Notre méthode sera interprétée comme une méthode de décomposition de domaines, ce qui permettra son étude de convergence. Les avantages de cette méthode résident dans la conservation de la structure creuse de la matrice éléments finis et la possiblilité de la factoriser une fois pour toutes au cours des itérations.

ABSTRACT. The aim of this paper is to study some iterative methods, based on the domain decomposition approach to solve the acoustic harmonic wave propagation in an unbounded domain. We describe how our methodology applies to semi-infinite closed guides and to acoustic scattering problems. In both cases, we use some well-known transparent boundary conditions by imposing on a fictitious boundary a boundary condition by the means of a Fourier expansion. For numerical purposes, we propose an original algorithm based on a fixed-point technique applied to the problem set in the truncated domain. We will interprate this method as a domain decomposition solver which allows to state convergence results. The improvement brought by this method is a consequence of the sparsity preservation of the finite matrix system which is decomposed only once.

MOTS-CLÉS : Conditions aux limites transparentes, décomposition de domaines, équation de Helmholtz.

KEYWORDS : Transparent boundary conditions, domain decomposition, Helmholtz equation.

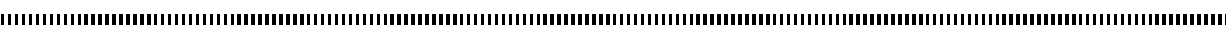

2006 Volume 4, pages 1-23 - ARIMA

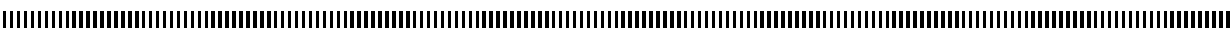

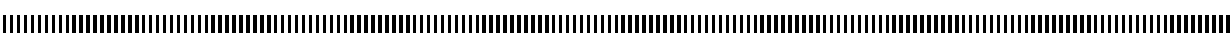

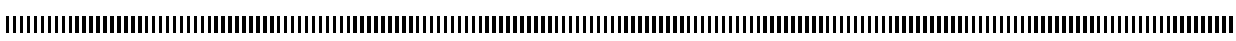

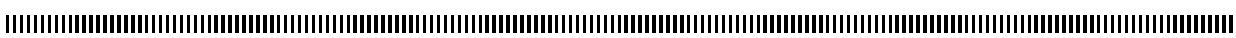

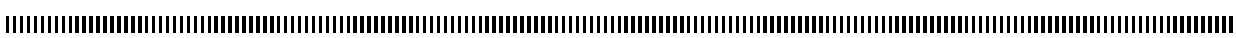

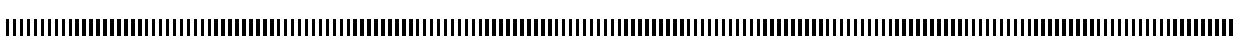

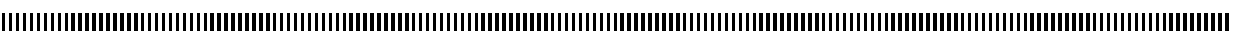

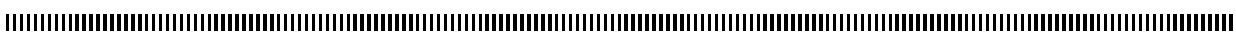

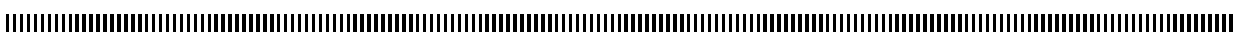




\section{Introduction}

We consider first time-harmonic scalar wave propagation in an unbounded waveguide. The most direct and natural approach consists in using a truncation of the infinite waveguide by a fictitious boundary (denoted $\Sigma$ ) on which an artificial boundary condition is imposed. Different boundary conditions can be found in the literature, we shall focus on a nonstandard one, consisting in writing down explicitely the Dirichlet-to-Neuman operator $T$, which is made possible by a variables separation. We reduce the initial problem to an equivalent one, set in a bounded domain, with exact transparent boundary condition on the fictitious boundary $\Sigma$. This method has been already proposed for several problems (Lenoir-Tounsi [12], Bonnet [4], Bonnet-Starling [6], Bonnet-Gmati [5], Ferreira [10], Cutzach-Luneville[9], Mahé [13], Killer-Givoli [11], Oberai-Malhotra-Pinsky [15], Razafiarivelo [17]). The continuous problem is then discretized by a finite element method. The numerical handling of the operator $T$ is not easy, because of its non-local character : the degrees of freedom on $\Sigma$ are coupled. This breaks the typical sparsity of the finite element matrix. We propose here a new algorithm based on a fixed-point technique applied to the problem set in the truncated domain. Although it is can be applied as well to three-dimensional problems, we illustrate it here in the two-dimensional case. Even though we only consider a Neumann boundary condition on the boundaries of the waveguide, the method we present and the techniques we formulate are also valid for Dirichlet and Robin-type boundary conditions.

In order to run the convergence analysis of our method, we interprate it as an iterative non-overlapping subdomain method. Our work is then connected to others in the same field, see A. Bendali and Y. Boubendir ([3], [7]). The difference lies in the fact that we impose that the interface between the subdomains is of a separable shape. This allows us to explicitely compute the solution of the second problem allowing the economy of solving a numerical problem in exterior domain. Moreover, we can state the convergence of the relaxed algorithm ([7]). However, in the present work the convergence analysis is carried out only on a rectangular semi-infinite waveguide and for a circular scatterer. This allows to understand the importance of the various modes of propagation, on the convergence phenomenon.

To explore the performances of such iterative methods, we focus on the overlapping domain decomposition. We will show that this method improves the convergence of the evanescent modes, but deteriorates that of some propagative modes. A Krylov method can then be used for the inversion. Even though the algorithm has been ran in the case of the waveguide, it can be extended to the case of acoustic scattering problems.

The outline of the paper is as follows. The mathematical background is given in section 1. Section 2 deals with the new algorithm based on a fixed-point method, and its interpretation as a non-overlapping domain decomposition method. We then present a va 
riant of this algorithm relaxing the transmission condition, and an overlapping domain decomposition method. In section 3, the convergence proofs are detailed in the simple case of a rectangular semi-infinite waveguide and for a scattering problem, the scatterer being a disk. In the final, we discuss some numerical experiments.

\subsection{The diffraction problem}

The method is described for both problems of the waveguide and scattering by a rigid bounded body. For the sake of clarity we start with the semi-infinite waveguide. The case of an infinite waveguide is a straightforward extension. For the case of a scattering problem, we discuss only the specific results.

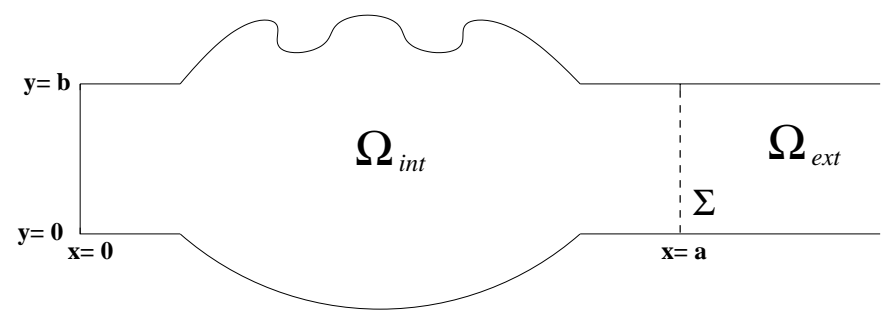

Figure 1. Geometry of the diffraction problem

Let $a$ and $b$ be two positive real numbers. We denote by $\Omega$ the bidimensional waveguide (Figure1). Then let $\Omega_{\text {int }}$ and $\Omega_{\text {ext }}$ be a partition of $\Omega, \Omega_{\text {int }}$ is bounded and $\Sigma=\left\{(x, y) \in \mathbb{R}^{2}\right.$ with $x=a$ and $\left.0<y<b\right\}$ is the common boundary to both subdomains. $\Omega_{\text {ext }}=\left\{(x, y) \in \mathbb{R}^{2}\right.$ with $x>a$ and $\left.0<y<b\right\}$ is the reduced exterior domain as shown in Figure 1, which is a rectangular waveguide.

Let $(x, y)$ be a generic point in $\Omega$. The waveguide is supposed to be submitted to an incident field $u_{i n}$. For example $u_{i n}=e^{-i k x}$ is a propagative plane wave in the direction $x<0$, where $k$ is the wavenumber. The governing equation for the diffracted field $u$ being the Helmholtz equation in the unbounded domain $\Omega$, with a Neumann boundary condition on $\partial \Omega$, which needs to be completed by a radiation condition (Cutzach-Luneville [9]) in order to select the outgoing modes (Razafierivelo [17]) :

$$
\left\{\begin{array}{cl}
u_{0}(y) & =O(1), \quad x \rightarrow+\infty \\
\forall m \in \mathbb{N}^{*}, \frac{d u_{m}}{d x}(y)-i k_{m} u_{m}(y) & =o(1), \quad x \rightarrow+\infty
\end{array}\right.
$$


where $u_{m}=\left(u, \varphi_{m}\right)_{0, \Sigma}$, the system $\left\{\varphi_{m}\right\}_{m \geq 0}$ is an orthonormal Hilbert basis of $\mathbb{L}^{2}(\Sigma)$, $(., .)_{0, \Sigma}$ the inner product in $\mathbb{L}^{2}(\Sigma)$ and the propagation constant $k_{m}$ related to the $m$-th mode is given by :

$$
k_{m}=\left\{\begin{array}{cc}
\left(k^{2}-\frac{m^{2} \pi^{2}}{b^{2}}\right)^{\frac{1}{2}}, & m<\frac{k b}{\pi} \\
i\left(\frac{m^{2} \pi^{2}}{b^{2}}-k^{2}\right)^{\frac{1}{2}}, & m \geq \frac{k b}{\pi}
\end{array}\right.
$$

For the diffraction problem by a rigid bounded obstacle $\omega$ in $\mathbb{R}^{2}, \Omega$ denotes the complement of $\bar{\omega}$ in $\mathbb{R}^{2}$. $\Omega_{\text {int }}$ and $\Omega_{\text {ext }}$ is a partition of $\Omega$. Their common boundary $\Sigma$ is a circle of radius $a$. For this second problem the radiation condition is the well known Sommerfeld condition : $\partial u / \partial r-i k u=o\left(1 / r^{1 / 2}\right), r \rightarrow+\infty$. Let $(P)$ be the problem set in $\Omega$, solved by the diffracted field $u$.

$$
(P)\left\{\begin{array}{l}
\text { Find } u \in \mathbb{H}_{l o c}^{1}(\Omega), \text { such that } \\
\Delta u+k^{2} u=0 \text { in } \Omega \\
\frac{\partial u}{\partial \nu}=f \text { on } \partial \Omega \\
+ \text { radiation condition, }
\end{array}\right.
$$

where $\mathbb{H}_{\text {loc }}^{1}(\Omega)$ is the space of functions that belong to $\mathbb{H}^{1}(\mathcal{D})$ for any open-bounded set $\mathcal{D}$ in $\Omega$ and $f=-\frac{\partial u_{i n}}{\partial \nu}$ arises from the incident wave $u_{i n}$. In the waveguide case, in order to simplify the presentation, $f$ is assumed to be compactly supported, i.e. $\operatorname{supp}(f) \subset$ $\partial \Omega_{i n t}$. It is the case of an incident plane wave $u_{i n}=e^{-i k x}$, which is independant of the coordinate $y$. We aim a finite element approximation, then, we shall introduce next the localized finite element method, that allows to reduce the computations to a bounded domain.

\subsection{A reduced problem}

The localized finite element method consists in truncating the initial domain $\Omega$ to the bounded one $\Omega_{\text {int }}$ and in using a transparent boundary condition on the fictitious boundary $\Sigma$, based on the modes of the rectangular waveguide. This condition is expressed in terms of the Dirichlet-to-Neuman operator, which is made possible by variables separation. 
The reduced differential problem to be solved can be stated as follows :

$$
\left(P_{\Sigma}\right)\left\{\begin{array}{rlrlrl}
\text { Find } u \in \mathbb{H}^{1} & \left(\Omega_{\text {int }}\right), \text { such that } \\
\Delta u+k^{2} u & = & 0 & \text { in } & \Omega_{\text {int }} \\
\frac{\partial u}{\partial \nu} & = & f & \text { on } & \partial \Omega_{i n t} \backslash \Sigma \\
\frac{\partial u}{\partial \nu} & = & T\left(u_{\mid \Sigma}\right) & \text { on } & \Sigma .
\end{array}\right.
$$

where $T$ is the mapping from $\mathbb{H}^{1 / 2}(\Sigma)$ on $\mathbb{H}^{-1 / 2}(\Sigma)$ defined by :

$$
T(\varphi)=\sum_{m \in \mathbb{N}} i k_{m}\left(\varphi, \varphi_{m}\right)_{0, \Sigma} \varphi_{m}
$$

$k_{m}$ were introduced in [2], and the orthonormal Hilbert basis $\left\{\varphi_{m}\right\}_{m \geq 0}$ is defined to be

$$
\varphi_{0}(y)=\sqrt{\frac{1}{b}} \text { and } \varphi_{m}(y)=\sqrt{\frac{2}{b}} \cos \left(\frac{m \pi}{b} y\right), \forall m \geq 1
$$

Remark 1 : For the waveguide, the resulting problem $\left(P_{\Sigma}\right)$ is well posed, except for at most a countable set of irregular frequencies ([17]). Moreover, it is proven in [17] that it is equivalent to $(P)$ (defined in section 1.1). Precisely, if $u$ is a solution of $(P)$ then $u_{\mid \Omega_{\text {int }}}$ is a solution of $\left(P_{\Sigma}\right)$. Conversely, if $\tilde{u}$ is a solution of $\left(P_{\Sigma}\right)$, then there is a unique extension of it, that solves $(P)$. This means that the last equation in problem $\left(P_{\Sigma}\right)$ is a perfect nonreflecting (or absorbing) boundary condition, which prevents the waves reflection on the artificial boundary, and can thus be considered as a radiation condition. For the scattering problem, $\left(P_{\Sigma}\right)$ is well posed and equivalent to $(P)$, in the sense already mentioned above.

For the scattering problem, the fictitious boundary $\Sigma$ is a circle with radius $a$, and the Dirichlet to Neumann Operator $T$ defined on $\Sigma$ is given by :

$$
T(\varphi)=\sum_{m \in \mathbb{Z}} \frac{k\left(H_{m}^{(1)}\right)^{\prime}(k a)}{H_{m}^{(1)}(k a)}\left(\varphi, \varphi_{m}\right)_{0, \Sigma} \varphi_{m}
$$

Here $H_{m}^{(1)}$ is the Hankel function of the first kind and $\left\{\varphi_{m}(\theta)=\frac{1}{\sqrt{2 \pi}} e^{i m \theta}\right\}_{m \in \mathbb{Z}}$ is a Hilbert Basis of $\mathbb{L}^{2}(\Sigma)$. The truncated problem can be put under the following variational form :

$$
\left\{\begin{array}{l}
\text { Find } u \in \mathbb{H}^{1}\left(\Omega_{i n t}\right), u \neq 0 \text { such that } \\
a(u, v)-c(u, v)=l(v), \quad \forall v \in \mathbb{H}^{1}\left(\Omega_{\text {int }}\right)
\end{array}\right.
$$


where the forms $a(.,),. c(.,$.$) and l($.$) are defined by:$

$$
\begin{aligned}
a(u, v) & =\int_{\Omega_{i n t}}\left(\nabla u \nabla \bar{v}-k^{2} u \bar{v}\right) d \Omega_{i n t} \\
c(u, v) & =<T u, v>_{-\frac{1}{2}, \frac{1}{2}, \Sigma} \\
l(v) & =\int_{\left(\partial \Omega_{i n t} \backslash \Sigma\right)} f \bar{v} d \gamma
\end{aligned}
$$

where the brackets $<., .>_{-\frac{1}{2}, \frac{1}{2}, \Sigma}$ denote the duality product between $\mathbb{H}^{-1 / 2}(\Sigma)$ and $\mathbb{H}^{1 / 2}(\Sigma)$.

The continuous problem $\left(P_{\Sigma}\right)$ is then discretized by a finite element method. We introduce the shape function $w_{\alpha}$ for each node $\alpha$ and $u_{\alpha}$ the approximation of the solution in the node $\alpha$. For numerical purposes, we use a truncation of the operator $T$ denoted by $T^{M}$, where $M$ is related to the number of terms. Then the linear system can be formulated as follows :

$$
(A-C) U=F
$$

In (5), we set $A=\left(a\left(w_{\alpha}, w_{\beta}\right)\right)_{\alpha, \beta}, F=\left(l\left(w_{\beta}\right)\right)_{\beta}, U=\left(u_{\alpha}\right)_{\alpha}$ and $C=\left(C_{\alpha, \beta}\right)_{\alpha, \beta}$ such that, $\forall(\alpha, \beta) \in \mathbb{N}^{2}$ :

$$
C_{\alpha, \beta}=\left(T^{M} w_{\alpha}, w_{\beta}\right)_{0, \Sigma}=\sum_{|m| \leq M} \mu_{m}\left(w_{\alpha}, \varphi_{m}\right)_{0, \Sigma}{\overline{\left(w_{\beta}, \varphi_{m}\right)_{0, \Sigma}}}_{0}
$$

where $\mu_{m}$ is equal to $i k_{m}$ for the waveguide and to $\left(k\left(H_{m}^{(1)}\right)^{\prime}(k a)\right) / H_{m}^{(1)}(k a)$ for the scattering problem.

Handling numerically the fictitious boundary condition may arise some trouble becomes of the non-local character. The local support of the shape functions induces a band structure of the stiffness matrix because there is no interaction between two nonneighbour nodes. However $C_{\alpha, \beta}$ is nonzero for any pair of nodes $\alpha$ and $\beta$ related to $\Sigma$, since the degrees of freedom are coupled together. The typical sparsity of finite element matrix is broken, the method that we propose consists in using a fixed-point method for $\left(P_{\Sigma}\right)$, in order to restore the sparsity of the obtained linear system at each iteration.

\section{A fixed-point algorithm}

The iterative algorithm we use and study is based on a fixed-point technique applied to the problem $\left(P_{\Sigma}\right)$ set in the truncated domain $\Omega_{\text {int }}$, with a transparent boundary condition on $\Sigma$. 


\subsection{A non-overlapping domain decomposition method}

The main point is to write, at each iteration, the boundary condition as follows : $\underline{\partial u^{n+1}}$

$\partial \nu$ ir on $\Sigma$, which ch resulting diffraction problem is not well-posed for a countable set of values of the wavenumber, due to the irregular frequencies of the domain $\Omega_{i n t}$. However, these singularities are not intrinsic to the original problem, which suggests to modify the boundary condition in order to overcome this difficulty. Let us introduce the following problem :

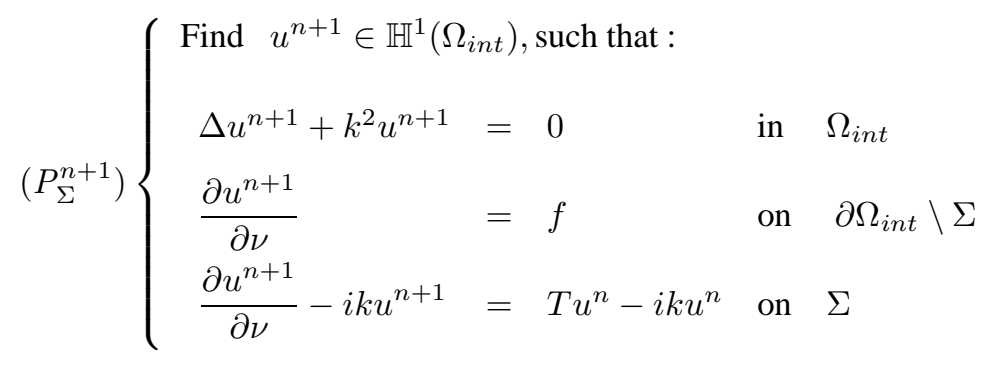

This problem with a Robin condition on $\Sigma$ is known to be well posed. By the means of a variational formulation, we show that the solution $\psi$ of the homogenous problem associated to $\left(P_{\Sigma}^{n+1}\right)$, satisfies $\frac{\partial \psi}{\partial \nu \mid \Sigma}=\psi_{\mid \Sigma}=0$. The theorem of unique continuation leads to $\psi=0$ on the domain $\Omega_{\text {int }}$. The algorithm is implemented under this form and can start from $u^{0}$ which satisfies $\frac{\partial u^{0}}{\partial \nu}-i k u^{0}=0$, on $\Sigma$.

To carry out the convergence analysis of the proposed method, we need an interpretation of it as an iterative non-overlapping subdomains method. As we will see the presented algorithm is nothing else than solving alternatly a sequence of problems set on the subdomains $\Omega_{i n t}$ and $\Omega_{\text {ext }}$. The boundary conditions are chosen iteratively by some appropriate transmission conditions between adjacent subdomains (Collino-Ghanemi-Joly [8], Benamou-Desprès [2], Boubendir [7]). The iterative procedure is defined using two sequences $\left(u^{n}\right)_{n \in \mathbb{N}}$ and $\left(v^{n}\right)_{n \in \mathbb{N}}$, solving the following problems :

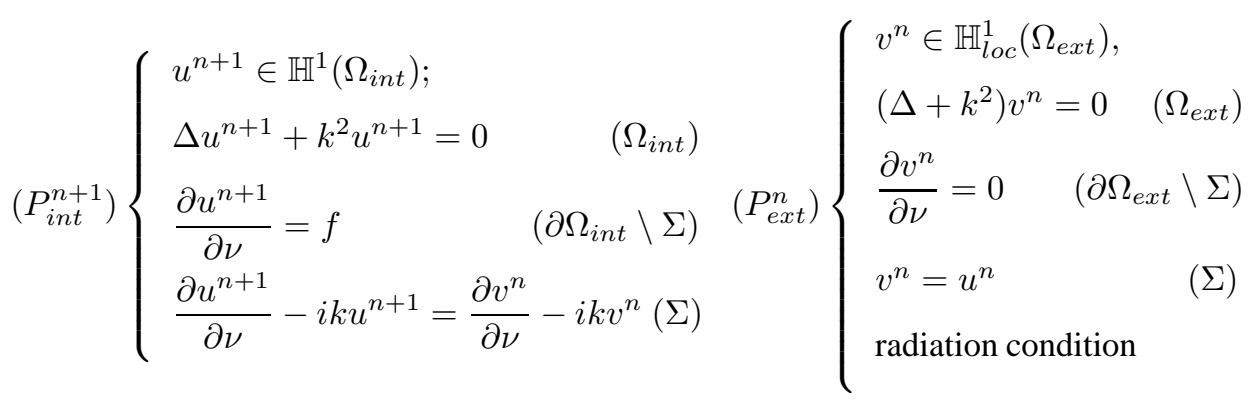


Let us recall that the assumption $\operatorname{supp}(f) \subset \partial \Omega_{\text {int }}$ induces a homogenous Neumann condition on $\left(\partial \Omega_{\text {ext }} \backslash \Sigma\right)$. The choice of a separable shape domain $\left(\Omega_{\text {ext }}\right)$ allows to give an explicit expression of the solution $v^{n}$ of $\left(P_{e x t}^{n}\right)$ which leads, from the definition of the operator $T$, to $\frac{\partial v^{n}}{\partial \nu}{ }_{\mid \Sigma}=T u^{n}$. From the second interface condition $v_{\mid \Sigma}^{n}=u_{\mid \Sigma}^{n}$, we can conclude that $\left(P_{\text {int }}^{n+1}\right)$ and $\left(P_{\Sigma}^{n+1}\right)$ are actually the same problem.

\section{Remark 2 : The Robin algorithm (or Després algorithm)}

We are able to prove that in some simple cases, the convergence of the previous problem $\left(P_{\Sigma}^{n+1}\right)$ works only for the incident propagative modes. A possible remedy is to modify the boundary conditions as a Robin boundary ones for both problems $\left(P_{e x t}^{n}\right)$ and $\left(P_{i n t}^{n}\right)$, as proposed in [2],[8],[16]. At each iteration, the functions $u_{n+1}$ and $v_{n+1}$ are connected by the following boundary conditions on $\Sigma$ :

$$
\frac{\partial v^{n+1}}{\partial \nu}+\lambda v^{n+1}=\frac{\partial u^{n}}{\partial \nu}+\lambda u^{n}, \frac{\partial u^{n+1}}{\partial \nu}-\lambda u^{n+1}=\frac{\partial v^{n+1}}{\partial \nu}-\lambda v^{n+1} .
$$

This can be rewritten, after substituting $v^{n+1}$, as follows :

$$
\left(\frac{\partial u^{n+1}}{\partial \nu}-\lambda u^{n+1}\right)=(T-\lambda I d)(T+\lambda I d)^{-1}\left(\frac{\partial u^{n}}{\partial \nu}+\lambda u^{n}\right) .
$$

Let us mention that the normal derivative of $u^{n+1}$ is related to the value of $u^{n+1}$ on $\Sigma$, by the boundary condition at the previous iteration, and need not to be evaluated numerically.

\section{Remark 3 : The relaxed algorithm}

The relaxed algorithm consists, in replacing the ingoing quantity $\left(\frac{\partial u^{n+1}}{\partial \nu}-i k u^{n+1}\right)$ by a linear combination of the same quantity at both steps $n$ and $n+1$. In other words, if we introduce a positive relaxation parameter $r \in] 0,1[$, the boundary condition can be rewritten as follows :

$$
\left(\frac{\partial u^{n+1}}{\partial \nu}-i k u^{n+1}\right)=r\left(T u^{n}-i k u^{n}\right)+(1-r)\left(\frac{\partial u^{n}}{\partial \nu}-i k u^{n}\right) \text { on } \Sigma .
$$

Notice that the original problem can be recovered by taking $r=1$.

We shall now illustrate how to extend the method we have presented to solve our problem with an alternative method inspired by the original overlapping Schwarz alternating method.

\subsection{An overlapping Schwarz alternating method}

To do so, we slightly modify the fictitious boundaries (cf. Figure 2 ) such that we keep the same definition for the domain $\Omega_{\text {int }}$, but $\Omega_{\text {ext }}=\left\{(x, y) \in \mathbb{R}^{2}\right.$ with $x>a_{0}$ and $0<$ 


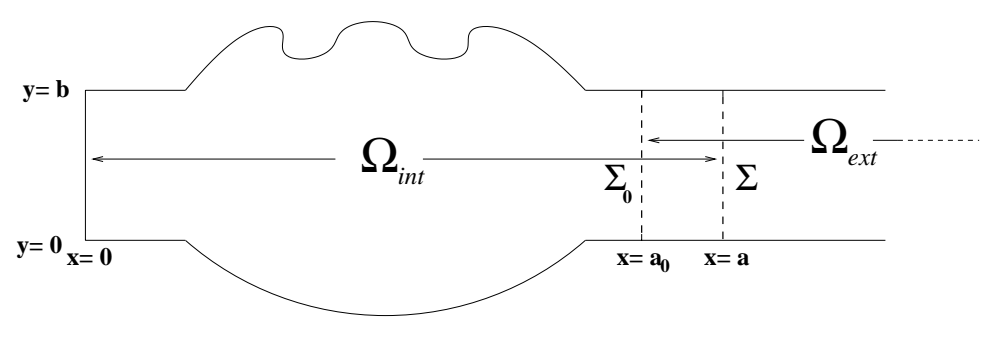

Figure 2. Overlapping decomposition domain.

$y<b\}$, for some real $a_{0}<a$, in such away that the overlapping domain is defined by $\Omega_{\text {ext }} \cap \Omega_{\text {int }}=\left\{(x, y) \in \mathbb{R}^{2}\right.$ with $a_{0}<x<a$ and $\left.0<y<b\right\}$.

For the scattering problem, $\Omega_{e x t}=\left\{(x, y) \in \mathbb{R}^{2}\right.$ with $\left.\left(x^{2}+y^{2}\right)^{\frac{1}{2}}>a_{0}\right\}$. The overlapping domain $\Omega_{e x t} \cap \Omega_{\text {int }}$ is the annular domain delimited by circles of radii $a$ and $a_{0}$.

We successively solve the Helmholtz equation in the two subdomains, keeping at each step the value on the internal boundaries as boundary condition for the next step : passing "Dirichlet" data from exterior subdomains to the other through the respective internal boundaries. The problems to be solved can be stated as :

$$
\left\{\begin{array} { l } 
{ u ^ { n + 1 } \in \mathbb { H } ^ { 1 } ( \Omega _ { \text { int } } ) ; } \\
{ \Delta u ^ { n + 1 } + k ^ { 2 } u ^ { n + 1 } = 0 } \\
{ \frac { \partial u ^ { n + 1 } } { \partial \nu } = f } \\
{ \frac { \partial u ^ { n + 1 } } { \partial \nu } - i k u ^ { n + 1 } = \frac { \partial v ^ { n } } { \partial \nu } - i k v ^ { n } ( \Sigma ) }
\end{array} \quad \left(\begin{array}{l}
\text { int } \backslash \Sigma) \\
\frac{\partial v^{n}}{\partial \nu}=0 \quad\left(\partial \Omega_{\text {ext }} \backslash \Sigma_{0}\right) \\
v^{n}=u^{n} \\
\text { Radiation condition }
\end{array} \quad\left(\Sigma_{0}\right)\right.\right.
$$

These problems do not arise any specific difficulty and could be treated by the usual techniques mentioned above. Observing that $v^{n}$ has an explicit expression, we can deduce the following on $\Sigma$ :

$$
\frac{\partial v^{n}}{\partial \nu}-i k v^{n}=\sum_{m \in \mathbb{N}}\left(i k_{m}-i k\right)\left(u_{\mid \Sigma_{0}}^{n}, \varphi_{m}\right)_{0, \Sigma_{0}} e^{i k_{m}\left(a-a_{0}\right)} \varphi_{m}
$$

Following the construction of operator $T$, we define the operator $\tilde{T}$ as a mapping from $H^{1 / 2}\left(\Sigma_{0}\right)$ into $H^{-1 / 2}(\Sigma)$ such that $\tilde{T}\left(u_{\mid \Sigma_{0}}^{n}\right)=\left(\frac{\partial v^{n}}{\partial \nu}-i k v^{n}\right)_{\mid \Sigma}$. The function $u^{n+1}$ in 
turn is a solution of a problem $\left(\tilde{P}_{\Sigma}^{n+1}\right)$ similar to the problem $\left(P_{\Sigma}^{n+1}\right)$ above, where we replace the operator $T$ by $\tilde{T}$, in the last equation on $\Sigma$.

\section{Analytical calculations and convergence study}

Some proofs of the convergence of the iterative subdomains algorithms are proposed for several standard elliptic equations. The convergence of these methods typically fails when applied to Hemholtz problem. In this context, to obtain strong convergence, Collino-Ghanemi-Joly ([8]), for problems in bounded domains and Boubendir ([7]), for unbounded domains impose non-local transmission conditions on the interface, and show some convergence results for the relaxed algorithms with local transmission conditions. Our aim is to establish convergence results of our algorithm for problems $\left(P_{\Sigma}^{n+1}\right),\left(\tilde{P}_{\Sigma}^{n+1}\right)$ and their associated relaxation. The proof is only based on a variable separation technique that is why we limit ourselves to the simple case of a semi-infinite rectangular waveguide and the diffraction by a disk of radius 1 . For the waveguide $\Omega_{\text {int }}=\{(x, y) \in$ $\mathbb{R}^{2}$ with $0<x<a$ and $\left.0<y<b\right\}, a_{0}$ being a real constant such that $a_{0}<a$, $\Omega_{e x t}=\left\{(x, y) \in \mathbb{R}^{2}\right.$ with $x>a$ and $\left.0<y<b\right\}$, for the non-overlapping method and $\Omega_{e x t}=\left\{(x, y) \in \mathbb{R}^{2}\right.$ with $x>a_{0}$ and $\left.0<y<b\right\}$ for the overlapping method. For the scattering problem $\Omega_{\text {int }}=\left\{(x, y) \in \mathbb{R}^{2}\right.$ with $\left.1<\left(x^{2}+y^{2}\right)^{\frac{1}{2}}<a\right\}$. $\Omega_{e x t}=\left\{(x, y) \in \mathbb{R}^{2}\right.$ with $\left.\left(x^{2}+y^{2}\right)^{\frac{1}{2}}>a\right\}$, for the non-overlapping method and $\Omega_{e x t}=\left\{(x, y) \in \mathbb{R}^{2}\right.$ with $\left.\left(x^{2}+y^{2}\right)^{\frac{1}{2}}>a_{0}\right\}$ for the overlapping method.

We denote by $e^{n}=\left(u^{n}-u\right)$ the error between the solution of problem $(P)$ and the solution of $\left(P_{i n t}^{n}\right)$ in $\Omega_{i n t}$, and $w^{n}=\left(v^{n}-u\right)$ the error between the solution of problem $(P)$ and the solution of $\left(P_{e x t}^{n}\right)$. Let $e_{m}^{n}=\left(e_{\mid \Sigma}^{n}, \varphi_{m}\right)_{0, \Sigma}$ and $w_{m}^{n}=\left(w_{\mid \Sigma}^{n}, \varphi_{m}\right)_{0, \Sigma}$ be the projections of their respective trace on the $\left\{\varphi_{m}\right\}_{m}$ basis defined above. Our goal is to evaluate the factor reduction between $e_{m}^{n}$ and $e_{m}^{n+1}$. 


\subsection{Convergence of the non-overlapping method}

The expression of $w^{n}(x, y)$ can be explicitly derived by technique of variables separation, indeed the solution of the following problem :

$$
\left\{\begin{array}{l}
w^{n} \in \mathbb{H}_{\text {loc }}^{1}\left(\Omega_{\text {ext }}\right), \\
\left(\Delta+k^{2}\right) w^{n}=0 \quad\left(\Omega_{\text {ext }}\right) \\
\frac{\partial w^{n}}{\partial \nu}=0 \quad\left(\partial \Omega_{\text {ext }} \backslash \Sigma\right) \\
w^{n}=e^{n} \quad(\Sigma) \\
\text { radiation condition }
\end{array}\right.
$$

is given by $w^{n}(x, y)=\sum_{m \in \mathbb{N}} e_{m}^{n} e^{i k_{m}(x-a)} \varphi_{m}(y)$. Taking into account that $e^{n+1}$ satisfies the Helmholtz equation in $\Omega_{\text {int }}$ and a homogenous Neumann boundary condition at $y=0$ and $y=b$, we obtain the following expression :

$$
e^{n+1}(x, y)=\sum_{m \in \mathbb{N}}\left(\gamma_{m} e^{i k_{m} x}+\xi_{m} e^{-i k_{m} x}\right) \varphi_{m}(y) .
$$

The homogenous Neumann boundary condition at $x=0$ leads to $\xi_{m}=\gamma_{m}$. On the other hand $e^{n+1}$ satisfies on $\Sigma$ the following boundary condition :

$$
\frac{\partial e^{n+1}}{\partial \nu}-i k e^{n+1}=\frac{\partial w^{n}}{\partial \nu}-i k w^{n}
$$

which can be rewritten as

$$
\begin{aligned}
\sum_{m \in \mathbb{N}} \gamma_{m}\left(i k_{m}\left(e^{i k_{m} a}-e^{-i k_{m} a}\right)\right. & \left.-i k\left(e^{i k_{m} a}+e^{-k_{m} a}\right)\right) \varphi_{m}(y) \\
& =\sum_{m \in \mathbb{N}} e_{m}^{n}\left(i k_{m}-i k\right) \varphi_{m}(y) .
\end{aligned}
$$

we derive that :

$$
\gamma_{m}=\frac{k_{m}-k}{k_{m}\left(e^{i k_{m} a}-e^{-i k_{m} a}\right)-k\left(e^{i k_{m} a}+e^{-k_{m} a}\right)} e_{m}^{n}
$$

From equation 9 we have the identity $e_{m}^{n+1}=\gamma_{m}\left(e^{i k_{m} a}+e^{-i k_{m} a}\right)$. We finally conclude that

$$
e_{m}^{n+1}(x, y)=\lambda_{m} e_{m}^{n}(x, y) \forall m, n \in \mathbb{N}
$$


where the reduction factor $\lambda_{m}$ is given by :

$$
\lambda_{m}=\frac{\left(k_{m}-k\right)}{i k_{m} \operatorname{tg}\left(k_{m} a\right)-k} \forall m \in \mathbb{N} .
$$

We immediately have : $\left|\lambda_{m}\right|<1$ if $m<k b / \pi$, and : $\left|\lambda_{m}\right| \geq 1$ if $m \geq k b / \pi$. This is enough to prove that the convergence mechanism of our fixed-point algorithm works only for the propagative modes. Of course the next step in the analysis consists in studying the behaviour of the relaxed problem. The convergence rate $\lambda_{m, r}$ is linked to the previous one $\lambda_{m}$ by :

$$
\lambda_{m, r}=(1-r)+r \lambda_{m} .
$$

To establish the convergence, all the $\left|\lambda_{m, r}\right|$ must be less than 1 . Taking into account the $\left|\lambda_{m}\right|$ behavior, us we see it on figure 3 , we can distinguish two cases :

(1) If $\mathcal{R} e\left(\lambda_{m}\right) \geq 1$, for all $\left.r \in\right] 0,1\left[,\left|\lambda_{m, r}\right|>1\right.$.

(2) If $\mathcal{R} e\left(\lambda_{m}\right)<1$, there exists $\left.r \in\right] 0,1\left[\right.$, such that $\left|\lambda_{m, r}\right|<1$

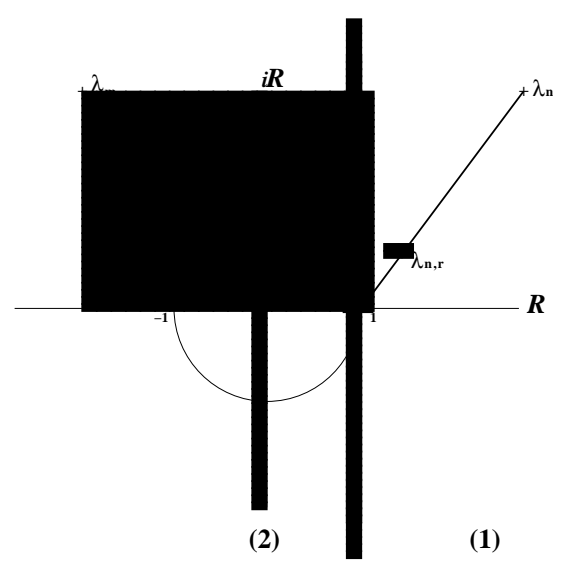

\section{Figure 3.}

It is clear that for the propagative modes, we have $\mathcal{R} e\left(\lambda_{m}\right)<1$ and for the evanescent modes $k_{m}$ is imaginary equal to $i \kappa_{m}$, such that $\kappa_{m}=\sqrt{\frac{m^{2} \pi^{2}}{b^{2}}-k^{2}}$, for which we have by easy calculations :

$$
\mathcal{R} e\left(\lambda_{m}\right)=\frac{k^{2}+\kappa_{m}^{2} \operatorname{th}\left(-\kappa_{m} a\right)}{k^{2}+\kappa_{m}^{2} \operatorname{th}^{2}\left(-\kappa_{m} a\right)}<1
$$

and since $\delta_{m}$ defined by $\delta_{m}=2\left(\operatorname{th}\left(-\kappa_{m} a\right)\right) /\left(\operatorname{th}\left(-\kappa_{m} a\right)-1\right)$ is a strictly increasing sequence, it is enough to prove the existence of $\delta=\inf _{m>(k b / \pi)} \delta_{m}$, such that for all $(0<$ 
$r \leq \delta),\left|\lambda_{m, r}\right|<1$. If $m=k b / \pi, k_{m}=0$, since $\lambda_{m}=1$, we obtain $\lambda_{m, r}=1$. The above analysis has shown that, there is at most one mode which is not affected by the relaxation method, namely $m=k b / \pi$. For all the other modes, if $r \in] 0, \delta]$, the reduction factor of the relaxed iterative method satisfies $\left|\lambda_{m, r}\right|<1$, what insures the convergence. Indeed, if we consider $\lambda_{m, r}$ as a function of $\mathrm{r}$ on $\left.] 0, \delta\right]$, we can show from its expression, that for each $m<k b / \pi$ (propagative modes), $\lambda_{m, r}$ is a decreasing function and the optimal value is then obtained for $r=\delta$. For each $m>k b / \pi$ (evanescent modes), $\lambda_{m, r}$ is a decreasing function of $r$ since $r<r_{m}$, and is increasing for $r>r_{m}$. The minimum of $\lambda_{m, r}$ is clearly reached for $r=r_{m}$. The expression of $r_{m}=\frac{t h\left(\kappa_{m} a\right)}{1+t h\left(\kappa_{m} a\right)}$ shows that its limit is $\frac{1}{2}$ when $m$ tends to $+\infty$.
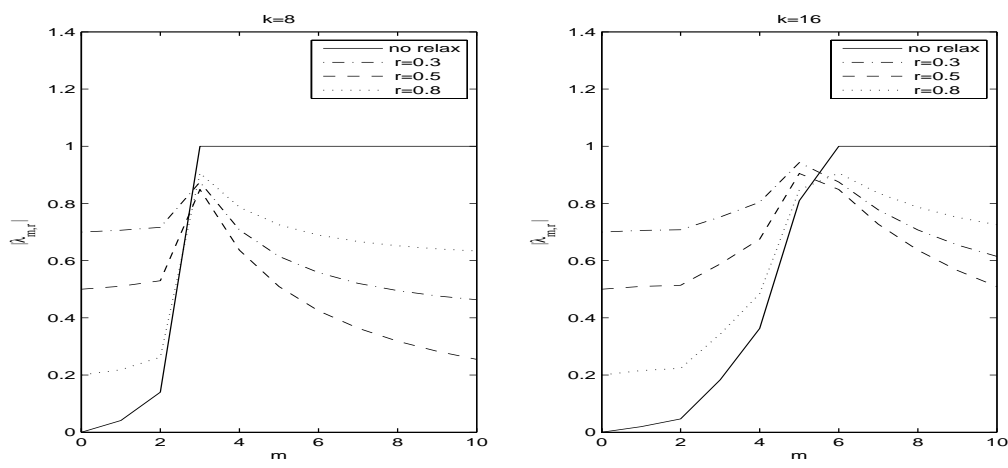

Figure 4. The modulus of the convergence rate $\lambda_{m, r}, a=1, b=1$

\section{Remark 4 : The Robin algorithm}

Using the Robin method ([7], [2],[8],[16]) defined above with $\lambda=i k$, leads to the following convergence rate :

$$
\lambda_{m}=\frac{\left(k_{m}-k\right)\left[i k_{m} \operatorname{tg}\left(k_{m} a\right)+k\right]}{\left(k_{m}+k\right)\left[i k_{m} \operatorname{tg}\left(k_{m} a\right)-k\right]} \forall m \in \mathbb{N} .
$$

We immediately obtain : $\left|\lambda_{m}\right|<1$ if $m<(k b / \pi)$, and : $\left|\lambda_{m}\right|=1$ if $m \geq(k b / \pi)$. This is sufficient for the convergence of the relaxed algorithm for all the modes, for any value of the relaxation parameter in $] 0,1\left[\right.$. Us in the last case, for propagative modes, $\lambda_{m, r}$ is a decreasing function of $r$ in $] 0,1]$. The optimal parameter is $r=1$, in other words the case without relaxation. On the other hand, for evanescent modes, $\lambda_{m, r}$ is a decreasing function of $r$ since $r<\frac{1}{2}$, and is increasing for $r>\frac{1}{2}$. The minimum of $\lambda_{m, r}$ is reached for $r=\frac{1}{2}$, for all the evanescent modes. 
For the scattering problem we have :

$$
\lambda_{m}=\frac{\frac{\left(H_{m}^{(1)}\right)^{\prime}(k a)}{H_{m}^{(1)}(k a)}-i}{\left(\frac{\left(J_{m}^{\prime}(k a)-\frac{J_{m}^{\prime}(k)}{Y_{m}^{\prime}(k)} Y_{m}^{\prime}(k a)\right)}{\left(J_{m}(k a)-\frac{J_{m}^{\prime}(k)}{Y_{m}^{\prime}(k)} Y_{m}(k a)\right)}-i\right)}
$$

The estimations of Bessel's functions does not allow us to treat all the modes, but gives us informations about the behaviour of $\left|\lambda_{m}\right|$ on well-defined parts describing these modes. Using an asymptotic expansion of Bessel functions ([1]), it is proven that $\left|\lambda_{m}\right| \simeq 0$ if $m \ll k$ and $\left|\lambda_{m}\right| \simeq 1$ if $m \gg k a$.

While we obtain the convergence of the algorithm for small values of $m(m \ll k a)$ which represent the propagative modes, the evanescent modes do not converge. As will be see, it is not a trivial matter to figure out exactly the $\left|\lambda_{m}\right|$ behaviour and this is the reason why a modal study is needed. An asymptotic study can show that $\lambda_{m}$ converges to 1 if $m \gg k a$ and is close to zero if $m \ll k a$.

Similarly to the semi-infinite waveguide, an appropriate choice of the relaxation parameter $r \in] 0,1$, permits us to overcome the convergence difficulty as can be observed in figure 5 .
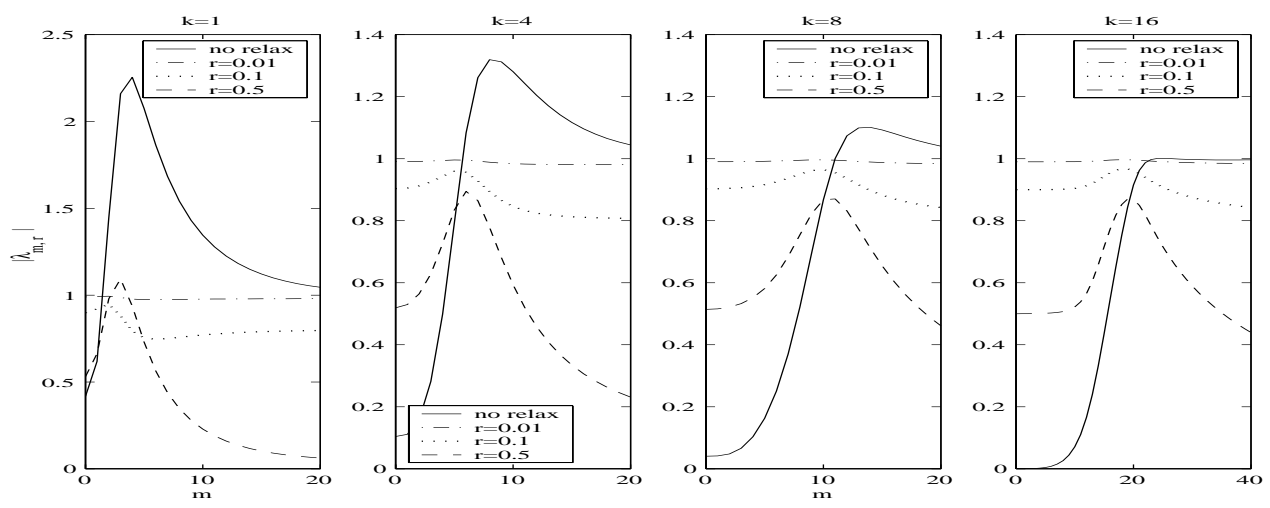

Figure 5. The $\lambda_{m, r}$ modulus 


\subsection{Convergence of the overlapping method}

Again for simplicity and whenever no risk of confusion may occur, we denote by $\lambda_{m}$ the rate of convergence of the overlapping alternating method. Hence, $e_{m}^{n+1}=\lambda_{m} e_{m}^{n}$, such that we obtain for the waveguide problem,

$$
\lambda_{m}=\frac{1+e^{-2 i k_{m} a_{0}}}{1-\frac{k_{m}+k}{k_{m}-k} e^{-2 i k_{m} a}}
$$

The same discussion as above, allows to check that :

1) if $m=k b / \pi,\left|\lambda_{m}\right|=1$.

2) We have that $\left|\lambda_{m}\right|<1$ for all $m<m_{1}$ for some $m_{1} \in \mathbb{N}$.

3) We have that $\left|\lambda_{m}\right|<1$ for all $m>m_{2}$ for some $m_{2} \in \mathbb{N}$.

The convergence is not guaranteed without an additional mechanism like a relaxation. For a finite number of modes, the reduction factor $\lambda_{m}$ is bigger than 1 . The advantage of the overlapping method, is that coefficients $\lambda_{m}$ are vanishing when $m$ goes to infinity. Then, the Steklov-Poincaré interface equation ([16]) can be solved efficiently by a Krylov method.

For the scattering problem the coefficients $\lambda_{m}$ is expressed as :

$$
\lambda_{m}=\frac{\left(\frac{\left[\left(H_{m}^{(1)}\right)^{\prime}(k a)-i H_{m}^{(1)}(k a)\right]\left[J_{m}\left(k a_{0}\right)-\frac{J_{m}^{\prime}(k)}{Y_{m}^{\prime}(k)} Y_{m}\left(k a_{0}\right)\right]}{H_{m}^{(1)}\left(k a_{0}\right)}\right)}{\left(J_{m}^{\prime}(k a)-\frac{J_{m}^{\prime}(k)}{Y_{m}^{\prime}(k)} Y_{m}^{\prime}(k a)\right)-i\left(J_{m}(k a)-\frac{J_{m}^{\prime}(k)}{Y_{m}^{\prime}(k)} Y_{m}(k a)\right)}
$$

The asymptotic expansion (cf. [1], [7]) fields that $\left|\lambda_{m}\right| \simeq 0$ if $m \ll k a$. On the other hand, if $m$ is large enough $(m \gg k a)$, such is the case for an evanescent mode, then $\left|\lambda_{m}\right| \simeq 0$.

To show that the overlapping method performs efficiently, for the evanescent modes, we set the artificial boundary radius to $a=2$, and we aim a comparison of the $\left|\lambda_{m}\right|$ behaviour. We consider various values of $k$ and we focus on three overlaps corresponding to $a_{0}=1.25,1.5$ and 1.75. In figure 6 the plots for $\log _{10}\left(\left|\lambda_{m}\right|\right)$ show the effect of the overlap for large $m$ and are in good agreement with the theoritical predictions.

Here also, the convergence can be accelerated by a relaxation procedure. 

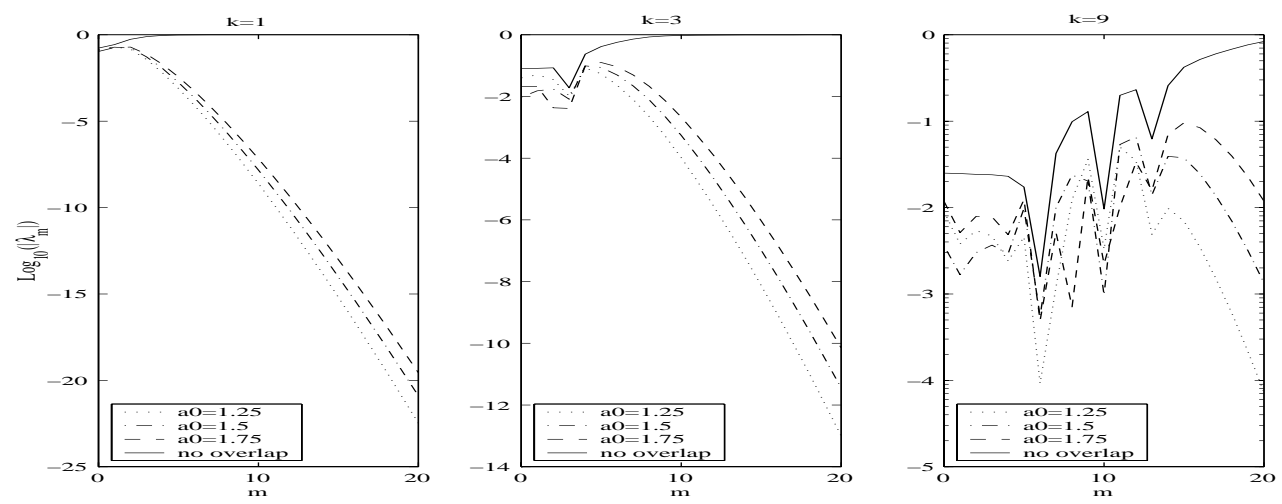

Figure 6. The $\log _{10} \lambda_{m}$ modulus

\section{Numerical results :}

The numerical implementation of the above methods is achieved in the numerical program Mélina developed by D. Martin [14]. It's an open collection of Fortran libraries for the solution of partial differential problems by finite element method. In all cases, we discritize the computational domain by $P 2$ finite elements, apply the method proposed in this paper, with or without relaxation parameter. Unless otherwise specified, solve the resulting algebraic system of equations by taking a truncation order $M=10$ of the operator $T$. As stated earlier, we solve the system of equation $(A-C) U=F$ to compute such a discrete solution. We compute the sequence solution $U^{n}$ by solving alternately the equation :

$$
\left(A-i k M_{\Sigma}\right) U^{n+1}=F+\left(C-i k M_{\Sigma}\right) U^{n}
$$

where $M_{\Sigma}$ is the mass matrix defined on $\Sigma$ and the initial guess has been chosen as $U^{0}=$ 0 . Futhermore, to investigate the convergence of the algorithm, we monitor the accuracy of all the cases by computing for each wavenumber $k$ the relative error value $\frac{\left\|U^{n}-U_{e x}\right\|_{2}}{\left\|U_{e x}\right\|_{2}}$ and the relative residual $\frac{\left\|(A-C) U^{n}-F\right\|_{2}}{\left\|U^{n}\right\|_{2}}$, where $U_{e x}$ is the exact solution and $\|\cdot\|_{2}$ is the discrete quadratic norm on $\Omega_{\text {int }}$.

\subsection{The rectangular waveguide}

In this section, we perform numerical experiments, for two-dimensional semi-infinite rectangular waveguide $\left.\Omega=\mathbb{R}^{+} \times\right] 0,1\left[\right.$. The computations are made on the domain $\Omega_{\text {int }}=$ ] $0,1[\times] 0,1[$. 


\subsubsection{No overlapping case}

We consider first an incident wave corresponding to the second propagative mode, $u_{i n}=\sqrt{2} e^{i \sqrt{k^{2}-\pi^{2}}(x-1)} \cos (\pi y)$. The mesh resolution is fixed to 10 elements per wavelength. We focus on the values of the wavenumber $k=5, k=10$ and $k=20$. In these cases we have respectively two, four and seven propagative modes. We show in figure 7 the relative error and the residual in $\Omega_{\text {int }}$. The results illustrate the accuracy of the method.
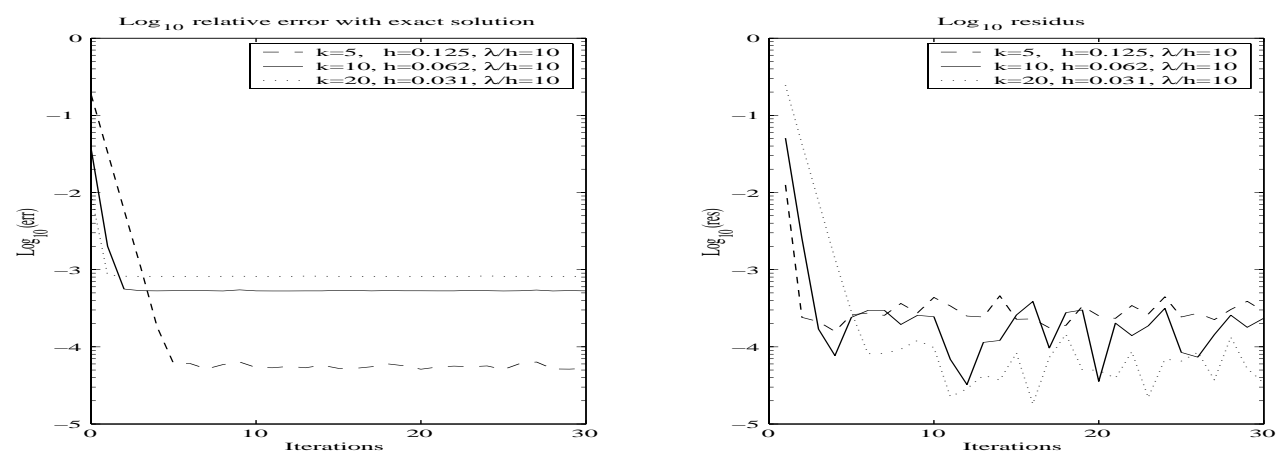

Figure 7. Propagative incident waves.
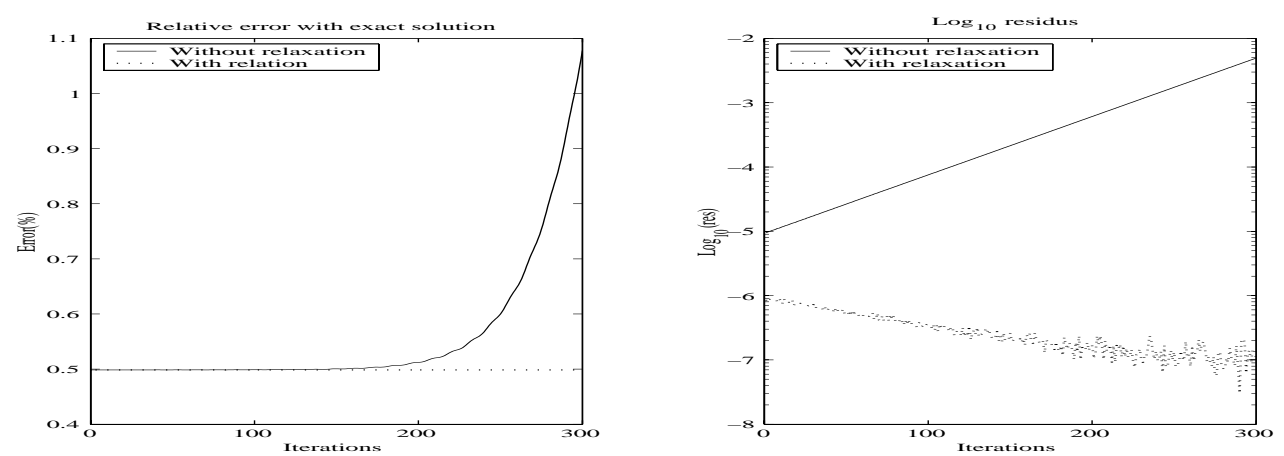

Figure 8. Evanescent incident wave, $k=3, r=0.1$

In the second simulation, we investigate the influence of the relaxation on the convergence of the method. We consider an evanescent incident mode corresponding to $u_{\text {in }}=$ $\sqrt{2} e^{-\sqrt{25 \pi^{2}-k^{2}}(x-1)} \cos (5 \pi y)$. In figure 8 , we choose $k=3, r=0.1$, and we plot on the same picture for the mesh size $h=0.062$, corresponding to 33 elements per wavelength, 
the variations of the relative error and the residual with respect to the number of iterations. One observes that, for the method without relaxation, the error level is acceptable in the 150 first iterations, and deteriorates after 200 iterations. The introduction of a relaxation parameter improve the convergence process. In figure 9, we consider three different values of the relaxation parameter corresponding to $r=0.1,0.5$ and 0.9 . We consider the same evanescent incident wave us the previous case. The results illustrate that the convergence of the algorithm is not obtained for $r=0.9$.

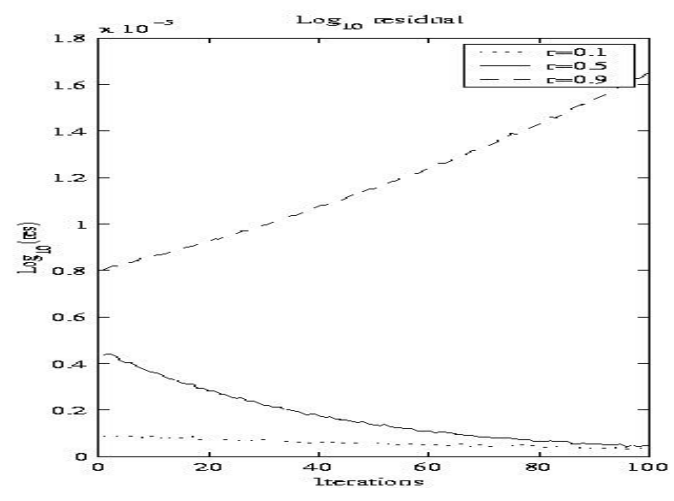

Figure 9. Evanescent incident wave, $k=3$.

\subsubsection{Overlapping case}
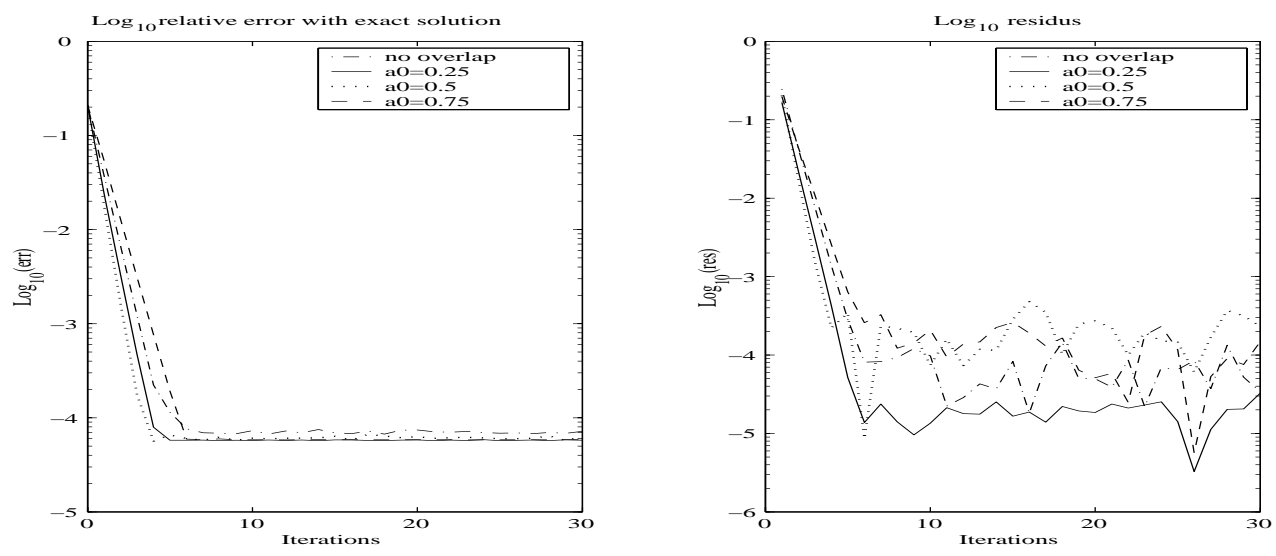

Figure 10. Propagative incident wave, $k=5$. 
We consider the same computational domain denoted by $\Omega_{\text {int }}=10,1[\times] 0,1[$ and we denote by $\left.\Omega_{2}=\right] a_{0}, 1[\times] 0,1[$ the overlapping area. We consider different values of the parameter $a_{0}$ (respectively $0.25,0.5$ and 0.75 ).

For the first simulation, the incident wave is the first propagative mode in the waveguide corresponding to $u_{i n}=\sqrt{2} e^{i \sqrt{k^{2}-\pi^{2}}(x-1)} \cos (\pi y)$. We fix the wavenumber $k=5$ and the mesh size $h=0.062$ corresponding to 20 elements per wavelength. We report in figure 10 the relative errors as well as their relative residual in the computational domain $\Omega_{\text {int }}$. For
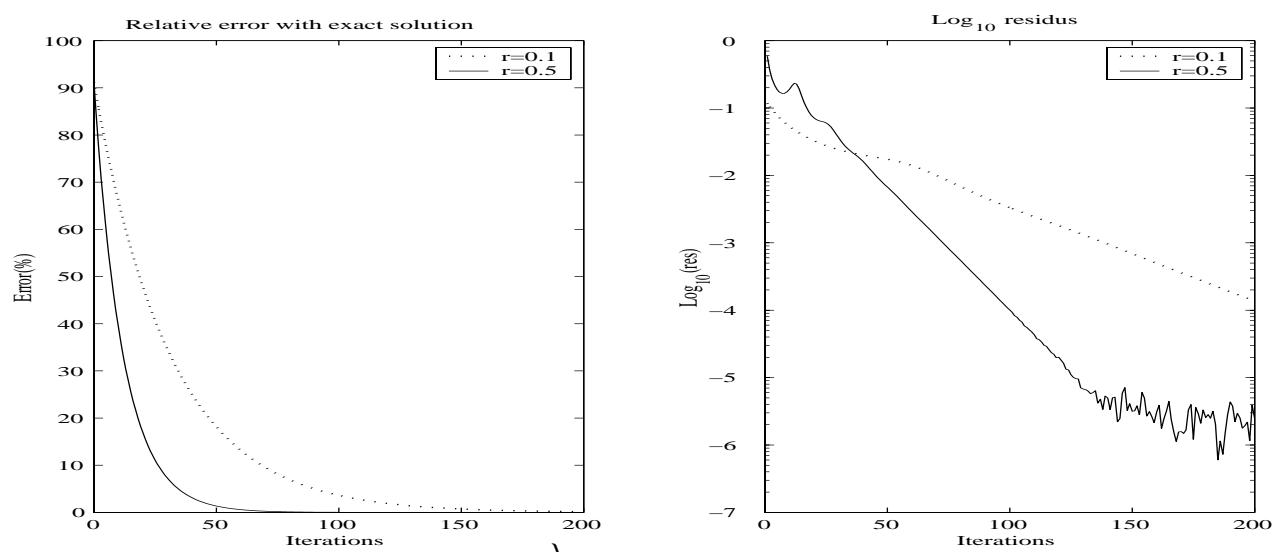

Figure 11. With relaxation, $k=3.25, \frac{\lambda}{h}=31$.
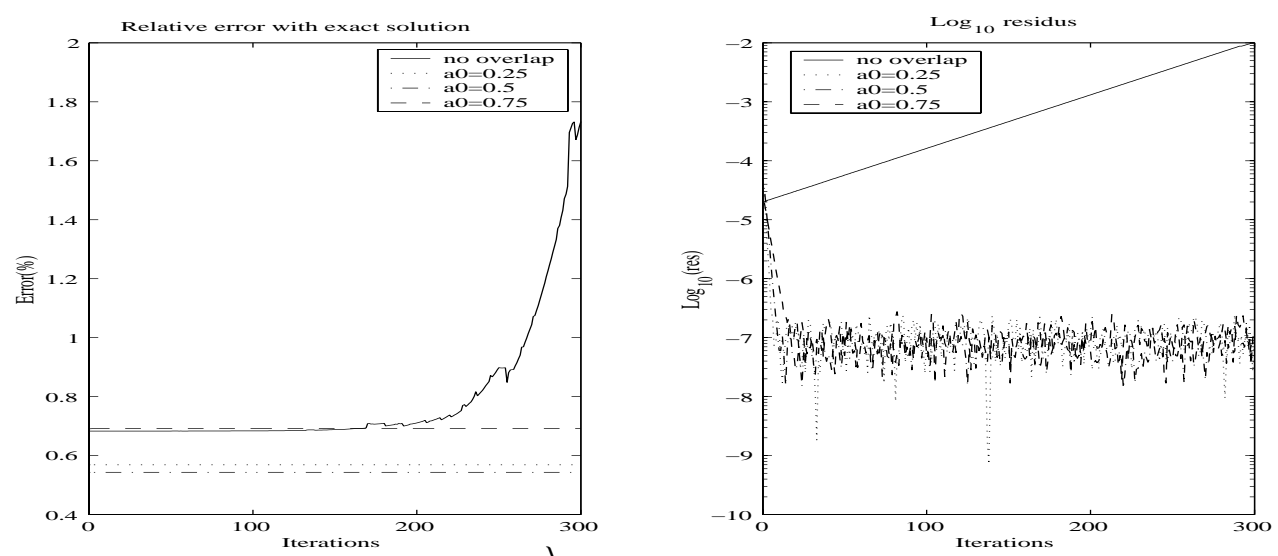

Figure 12. Evanescent wave, $k=3, \frac{\lambda}{h}=33$. 
the second simulation, we consider $k=3.25$ and the mesh size $h=0.062$. In this case we have two propagative modes in the wave guide, and the incident evanescent wave correspond to $m$ such that the condition $m<m_{1}=\sqrt{\frac{3}{4}} \frac{k}{\pi}$ is not satisfied. Figure 11 shows the convergence of the relaxed algorithm when we set the relaxation parameter to $r=0.1$ and $r=0.5$. For the case of evanescent incident mode $u_{\text {in }}=\sqrt{2} e^{-\sqrt{25 \pi^{2}-k^{2}}(x-1)} \cos (5 \pi y)$, we set $k=3$. In figure 12 we also compare the relative errors with respect to exact solution and the residual in the computational domain $\Omega_{i n t}$. Clearly the overlapping method shows a better behaviour than the non overlapping one.

\subsection{The scattering problem}
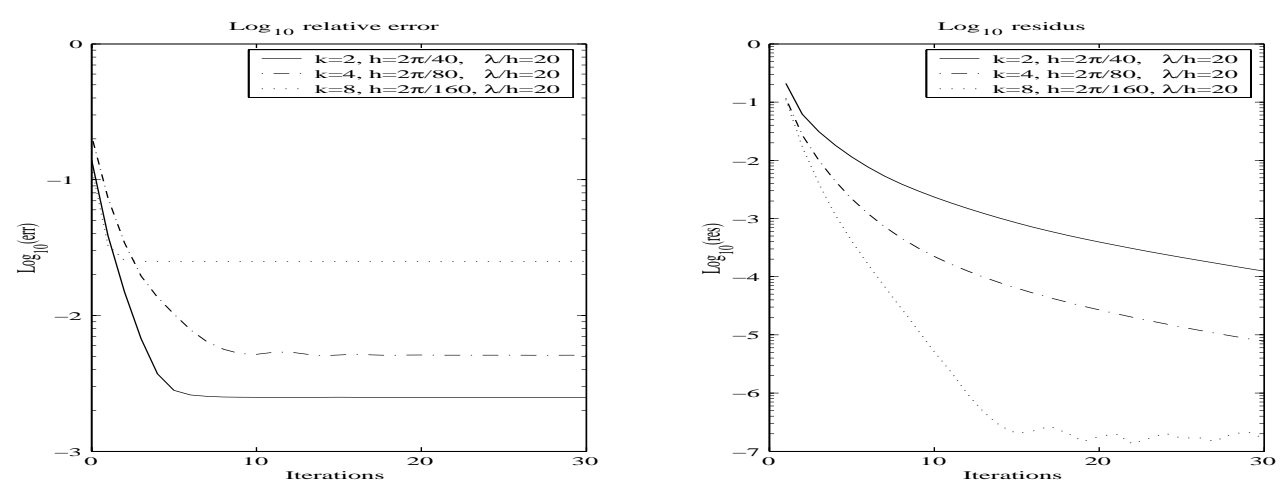

Figure 13. Incident plane mode $a=1.5$.

The objective is to highlight the possibilities of the proposed method for solving the acoustic scattering problem. We consider then a circular scattrer of radius 1 . Let $M=$ 10 be the truncation order of the Dirichlet-to-Neuman operator. We choose the exterior artificial boundary $\Sigma$ as a circle of radius $a$. We perform several computations for different wavenumbers, mesh sizes and for different positions of $\Sigma$, and compare our results to the analytical solution. The incident wave is planar given by $u_{i n}=e^{i k x}$ for the nonoverlapping case. The exact solution in the boundary value problem is :

$$
\begin{cases}-\frac{J_{1}(k)}{H_{1}^{1}(k)} H_{0}^{1}(k r) & \text { for } \quad m=0 \\ \frac{2 i^{m}\left(J_{m-1}(k)-J_{m+1}(k)\right)}{a\left(H_{m-1}^{1}(k)-H_{m+1}^{1}(k)\right)} H_{m}^{1}(k r) \cos (m \theta) & \text { for } \quad m \geq 1 .\end{cases}
$$

In figure 13 we set $a=1.5$ and we consider several wavenumbers corresponding to $k=2,4,8$, and generate meshes using 20 elements per wavelength. We notice that as 
the previous study, depending on the wavenumber $k$, the non-overlapping algorithm exhibits similar intervals of convergence. Next we set the artificial boundary $\Sigma$ at different
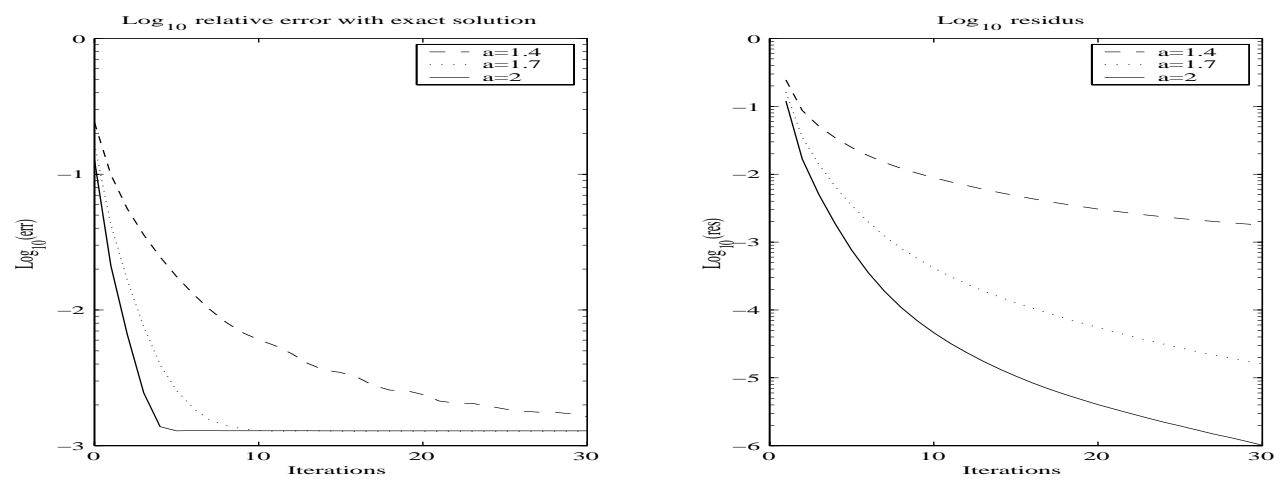

Figure 14. Incident plane mode, $k=2, \frac{\lambda}{h}=20$.
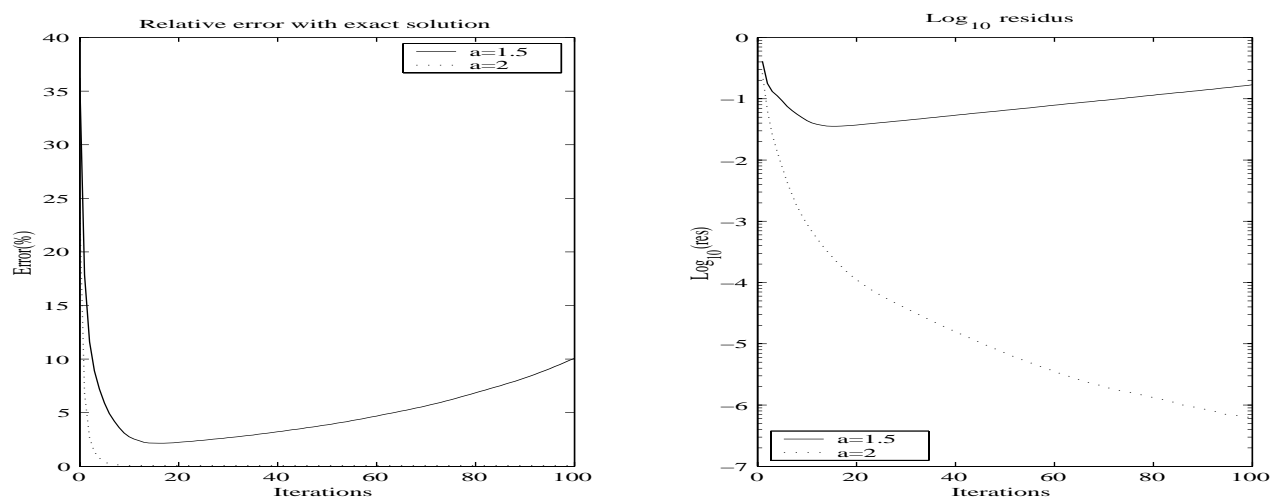

Figure 15. Incident plane mode, $k=1, \frac{\lambda}{h}=80$.

positions corresponding to $a=1.4,1.7$ and 2 and generate several meshes taking 20 elements by wavelengh. Notice that these meshes have been tailored to deliver a comparable accuracy. Indeed, using for each position of the artificial boundary its assigned mesh, we obtain the results summarized in figure 14 which show that the relative errors decrease with $a$ as expected. This is related to the fact that for $m \ll k a$, the convergence rate tends to 0 and for small values of $a$ taking into account evanescent modes which deteriorate the convergence process. In the following computation, for the incident plane wave, we show the role of the relaxation parameter. If we take $k=1$ and consider $a=1.5$ the algorithm 
diverges (Figure 15), while the relaxed algorithm converges for different values of the relaxation parameter (Figure 16). For the overlapping case, the numerical results show
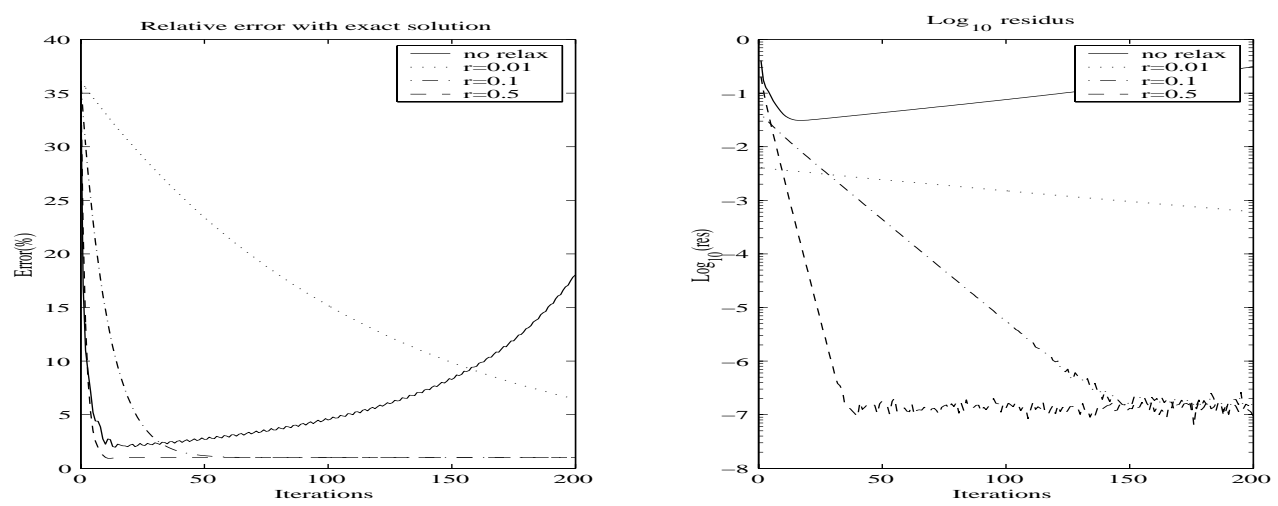

Figure 16. Incident plane mode, $k=1, \frac{\lambda}{h}=80, a=1.5$.

that, when the overlapping method is applied, and if the incident wave does not have components on some critical modes the convergence is faster than the non-overlapping algorithm.

Acknowledgements : We are pleased to address our special thanks to F. Ben Belgacem who is at the origin of this work and for his advices, and to D. Martin for providing us with his computing code MELINA. We also thank members of the project Ondes at INRIA for helpful discussions. This work is supported by the Tunisian Ministry for Scientific Research and Technology with the LAB-STI02 program.

\section{Bibliographie}

[1] Abramowitz M., Stegun I.A., « Handbook of mathematical functions », Dover Publications, New york, 1972.

[2] Benamou J.D., Després B., « A domain decomposition method for the Helmholtz equation and related optimal control problems », Comput. Phys., 136, 68-82, 1997.

[3] Bendali A., Boubendir Y., « Méthode de décomposition de domaine et éléments finis nodaux pour la résolution de l'équation d'Helmholtz », C. R. Acad. Sci., Paris, Sér. I Math., vol. 339, Issue 3, 229-234, August 2004.

[4] Bonnet A.S., DRissi D., GMATi N., « Mathematical analysis of the acoustic diffraction by a muffler containing perforated duct. », Math. Mod. Meth. in Appl. Sc. Vol.15, $n^{\circ}$ 7, 1059-1090, 2005. 
[5] Bonnet Bendhia A.S., Gmati N., « Spectral approximation of a boundary condition for an eigenvalue problem », SIAM J. NUMER. ANAL. Vol 32, n' 4, pp 1263-1279, August 1995.

[6] Bonnet-Bendhia A.S., Starling F., " Waves by electromagnetic gratings and nonuniqueness examples for the diffraction problem. » Mathematical Method in the Applied Sciences, Vol. 17, 305-338, 1994.

[7] BoubendiR Y., « Techniques de décomposition de domaine et méthode d'équations intégrales », Thèse de doctorat de l'Institut National des Sciences Appliquées de Toulouse, Juin 2002.

[8] Collino F. , Ghanemi S., Joly P., « Domain decomposition method for harmonic wave propagation : a general presentation », Computer methods in applied mechanics and engineering, $\mathrm{n}^{\circ} 184: 171-211,2000$.

[9] Cutzach P.M., Luneville E., « Diffraction d'ondes acoustiques par un guide semiinfini », C.R. Acad. Sci. Paris, Sér.I Math., 326, n 9, 1151-1154, 1998.

[10] FERreira A., « Etude numérique de quelques problèmes de diffractions d'ondes par des réseaux périodiques en dimension 2 », Thèse de doctorat de l'École polytechnique Paris, Novembre 1998.

[11] Killer J.B., Givoli D., « Exact non-reflecting boundary conditions », Journal of computational physics, Vol. 82, Issue 1, pp 172-192, 1989.

[12] Lenoir M. , Tounsi A., " The localized finite element method and its application to the two-dimensional sea-keeping problem », SIAM J. Numer. Anal. 25, pp :729-752, 1988.

[13] MAнÉ F., « Computing guided modes for an unbounded stratified medium in integrated optics », Mathematical Modelling and Numerical Analysis (M2AN), Vol. 35, N04, pp 799-824, 2001.

[14] Martin D., « Documentation MELINA », Rennes (1997) http ://www.maths.univrennes1.fr/ dmartin/melina/www/homepage.html.

[15] Oberai Assad A. , Malhotra M. , Pinsky Peter M., « On the implementation of the Dirichlet-to-Neumann radiation condition for iterative solution of the Helmholtz equation », Division of Mechanics and Computation, Departement of Mechanical Engineering, Standford University, California 94305, SUDMC Report no 97-05, December 1997.

[16] Quarteroni A., VAlli A. , « Domain decomposition methods for partial differential equations », Numerical mathematics and scientific computation, Oxford Univ. Press, 1999.

[17] RAZAFiARIVElo J., « Optimisation de la forme de transition entre guides éléctromagnétiques par une méthode intégrale d'éléments finis », Thèse de doctorat de l'université Pierre et Marie curie, 1996. 